\title{
La concepción relacional de la justicia y los límites de las reivindicaciones legítimas: la cooperación, la reciprocidad y la interacción ${ }^{1}$
}

\section{The Relational Conception of Justice and the Limits of Legitimate Claims: Cooperation, Reciprocity and Interaction}

\section{Francisco Blanco Brotons ${ }^{2}$ \\ Universidad Internacional de La Rioja (España)}

ORCID: https://orcid.org/0000-0001-8805-9637

Recibido: 03-02-2020

Aceptado: 30-04-2020

\section{Resumen}

Desde una concepción relacional de la justicia de inspiración rawlsiana, las ideas de la cooperación, la reciprocidad y la interacción han sido propuestas para interpretar el fundamento de las reivindicaciones legítimas de justicia distributiva y para restringir su ámbito de aplicación a sociedades políticas o Estados particulares. En este artículo se analizan los principales intentos de justificar esta restricción y se argumentará en contra de ellos. Se finalizará con la presentación de otra forma de interpretar la concepción relacional de la justicia que posibilita una aproximación crítica a las complejas injusticias con las que nos interpela nuestro mundo en globalización, según la cual, si cualquier elemento de

\footnotetext{
${ }^{1}$ Este artículo se ha elaborado en el marco de un proyecto de investigación financiado por el Ministerio de Ciencia, Innovación y Universidades: "Fronteras, Democracia y Justicia Global" (PGC2018-093656-B-100).

2 (francisco.blanco@unir.net) Doctor en Filosofía por la Universidad Complutense de Madrid. En la actualidad participa en el proyecto "Fronteras, democracia y justicia global. Argumentos filosóficos en torno a la emergencia de un espacio cosmopolita" del IFS-CSIC financiado por el Ministerio de Ciencia, Innovación y Universidades de España. Sus temas de investigación son la filosofía política y las teorías de la justicia contemporáneas, con especial atención a los problemas de justicia global. Algunas de sus publicaciones son: "La prioridad de la injusticia. El giro copernicano en teoría de la justicia", Filosofia Unisinos, vol.21, n.3, 2020, 277-285; "The Concept of No-man's-land as a Categorial Error with Meaning", Annals of the University of Bucharest. Philosophy Series, vol. 69, n.1, 2020, 83-100; "Expanding the Idea of Structural Injustice: Migrants and Global Justice", en Velasco J.C y La Barbera M.C, Challenging the Borders of Justice in the Age of Migrations, Cham: Springer, 2019, 117-138 y "La política del marco. Reescribiendo la injusticia de las fronteras de la ciudadanía", en Revista de filosofia, vol.42, n.2, 2017, 275-289.
} 
la estructura social impone relaciones evaluables desde la justicia, cualquiera de ellos puede ser un foco de injusticia, ya sea básico o no, formal o informal, estatal o trasnacional.

Palabras-clave: justicia global, estructura social, injusticia estructural, complejidad global, marcos de la justicia.

\begin{abstract}
From a rawlsian-inspired relational conception of justice, the ideas of cooperation, reciprocity and interaction have been proposed to interpret the grounds for legitimate claims to distributive justice and to restrict its scope of application to particular political societies or states. This article analyses the main attempts to justify this restriction and argues against them. The paper will conclude with the presentation of another way of interpreting the relational conception of justice that makes possible a critical approach to the complex injustices with which we are challenged by the current globalizing world, according to which, if any element of the social structure imposes relations that can be evaluated as just or not, any of them can be a focus of injustice, whether basic or not, formal or informal, national or transnational.
\end{abstract}

Key-words: global justice, social structure, structural injustice, global complexity, frames of justice.

Teoría de la justicia de Rawls ha influido más que ningún otro libro en los debates en torno a la teoría filosófica de la justicia desde su publicación (1971) hasta la actualidad. La profunda repercusión que tuvo esta obra ayudó a consolidar una interpretación general sobre qué es y de qué trata una teoría de la justicia que se sintetiza en esta idea: "el objeto primario de la justicia es la estructura básica de la sociedad» (Rawls 2014: 20). De acuerdo con esta idea, que instaura lo que denominaremos la "concepción relacional de la justicia", la justicia es una virtud de las estructuras sociales que median las relaciones entre los individuos. Esta concepción, en la que coinciden todos los autores que se analizarán en este artículo, es una interpretación general de qué es la justicia y cuáles son sus límites y, por lo tanto, es una cuestión previa a la deducción de principios concretos de justicia. Rawls, sin embargo, estaba más interesado en esta última cuestión, por lo que la primera, en especial en relación a los límites que esta idea imponía al alcance de la justicia y a los límites de las reivindicaciones legítimas, no recibió análisis sistemático. Como es sabido, él presuponía que el ámbito de aplicación de su teoría eran sociedades cerradas 
y autosuficientes regidas por una única estructura básica. Sólo a partir del final del siglo XX, cuando esta presuposición y en general el «nacionalismo metodológico" entraron abiertamente en crisis, los autores que se enmarcaban en la perspectiva relacional tuvieron que reflexionar explícitamente sobre la restricción de los principios de la justicia a algún ámbito específico.

La perspectiva relacional impone un límite muy preciso al fundamento de las reivindicaciones legítimas y al conjunto de personas que se deben justicia recíprocamente o frente a las que ciertas instituciones están obligadas a responder. Este conjunto de personas debe encontrarse relacionado por una estructura social relevante cuya injusticia pretende resolverse. Si dos personas no se encuentran relacionadas por ninguna estructura social, no se darán reivindicaciones legítimas de justicia entre ellas. Paralelamente, si se puede identificar alguna estructura social entre ellas, las reivindicaciones legítimas de justicia no serán de cualquier tipo o necesariamente iguales a las que se dan entre algún otro conjunto de personas, sino relativas a esa estructura, a lo que hace y cómo lo hace (Freeman 2006; Sangiovanni 2008). Desde esta concepción de la justicia, cobra una importancia fundamental qué interpretación se haga del tipo de relación que fundamenta la clase de reivindicaciones de justicia que se estén analizando. Según cual sea, las personas que se deben justicia mutuamente serán diferentes. Clarificar los criterios que hacen a determinadas relaciones estructurales relevantes para la justicia cobra un papel fundamental en los debates sobre justicia global.

Diversos autores han empleado esta perspectiva para justificar que las únicas instituciones sociales relevantes a las que se aplica los principios de la justicia social igualitaria son las que conforman los Estados soberanos, de modo que la justicia impondría demandas y obligaciones basadas en estos principios sólo a los conciudadanos de un mismo Estado y a sus instituciones nacionales. Según tales autores, sólo las relaciones bajo las que el Estado sitúa a sus ciudadanos dan pie a reclamaciones legítimas de justicia social. ¿Qué es lo que distingue estas relaciones de las que se establecen con agentes diferentes al propio Estado o con personas que no comparten una misma nacionalidad? Como veremos, a pesar de que algunos autores como Nagel llegan al extremo de afirmar que la justicia global sin un gobierno mundial es una quimera, lo que se discute en estos debates es más bien la existencia de un tipo particular de exigencias de justicia, en concreto, las relacionadas con la justicia distributiva igualitaria. El punto que estos autores más específicamente quieren rechazar es la extensión de los principios distributivos igualitarios que Rawls pensó para la estructura básica del Estado al orden global mediante algún tipo de posición original, sin atender a la especificidad de las relaciones establecidas en cada uno de estos niveles. En este artículo se analizarán algunos de los principales criterios formulados para evitar tal extensión, según han sido propuestos por 
Freeman, Sangiovanni y Abizadeh y Miller, respectivamente la cooperación, la reciprocidad y la interacción. Sin cuestionar la idea de que un contexto diferente requiere exigencias diferentes de justicia (Cohen y Sabel 2006: 148; Nagel 2008: 170; Sangiovanni 2007: 5), se defenderá aquí que ninguno de esos criterios puede restringir radicalmente la justicia al espacio del Estado soberano: ni la justicia distributiva igualitaria tiene sentido sólo en el interior de los Estados, ni fuera de estos hay sólo obligaciones de asistencia humanitaria. Se concluirá defendiendo una interpretación de la concepción relacional de la justicia capaz de responder a las complejas injusticias del actual mundo en globalización, según la cual, si cualquier elemento de la estructura social impone relaciones evaluables desde la justicia, cualquiera de ellos puede ser un foco de injusticia, ya sea básico o no, formal o informal, estatal o trasnacional.

\section{Freeman}

Freeman se propone defender que los principios de justicia distributiva planteados por Rawls sólo pueden aplicarse a las instituciones básicas de cada sociedad particular, mientras que a nivel global sólo existe la obligación de respetar los derechos humanos (Freeman 2006: 33-35). Esta diferencia se debe a que sólo en el primer contexto existe "cooperación social", y los principios de justicia distributiva están diseñados específicamente para tal tipo de relación social. Cooperación social no hace simplemente referencia a una acción coordinada para lograr determinados fines, algo que sin duda existe en las instituciones globales, sino a una cooperación realizada según términos equitativos. Esto quiere decir que las personas que cooperan en cada sociedad particular lo hacen según un sentido común de justicia y no simplemente guiados por su beneficio individual. Es la existencia de una "estructura básica de la sociedad" (id. 38) que mantiene unas condiciones justas de trasfondo lo que hace posible una cooperación social así entendida. Dentro de esta estructura básica, Freeman resalta la importancia de las instituciones políticas: "what makes possible the incredibly complicated system of legal norms that underlie economic production, exchange, and consumption is a unified political system that specifies these norms and revises them to meet changing conditions" (id., 39). A nivel global no existe cooperación social en el sentido requerido porque no hay una estructura básica comparable a la del marco nacional ni unas estructuras políticas capaces de ejercer una autoridad independiente. Los principios de justicia distributiva sirven para regular la estructura básica, por lo que la conclusión de Freeman es que este tipo de justicia no es exigible en el marco global. A continuación, se expondrán algunas objeciones a este planteamiento. 
Según Freeman, la diferencia fundamental entre el contexto global y el nacional es que sólo en este existe lo que él ha denominado cooperación social. Ésta no designa simplemente un sistema de interacción coordinado para alcanzar algún fin, sino un sistema de interacción justo o equitativo. Lo que diferencia un sistema de otro es que en éste hay una estructura básica que no sólo contiene unas normas reguladoras, sino también unas instituciones políticas con autoridad para definir tales normas y adaptarlas a las circunstancias de modo que se mantengan las condiciones justas de trasfondo. Según la terminología de Freeman, que a nivel supra-nacional sólo exista interacción social pero no cooperación social significa simplemente que aquí las interacciones sociales no se realizan en términos equitativos. Pero entonces, lo que la propuesta de Freeman hace es afirmar que los principios de justicia distributiva sólo son aplicables en los contextos donde ya de hecho existe cooperación social, es decir, interacción justa. Inversamente, estos principios de justicia no se aplican a nivel global precisamente porque este nivel es ya de hecho injusto. Estamos ante lo que denominaré una "perversión normativa»: la idea de que debido precisamente a que una situación no es justa, la reivindicación de que lo sea es ilegítima. Sin duda, en el nivel global se dan circunstancias de dominación o explotación que hacen difícil ver en ellas una beneficiosa relación de cooperación, pero de este hecho nuestra conclusión no debe ser que en tal nivel no tienen sentido las reivindicaciones de justicia, sino por el contrario que especialmente allí deben ser urgentemente atendidas.

Por otra parte, si la estructura básica es un medio para cumplir con el objetivo de realizar la justicia, como se desprende de la propuesta de Freeman, su existencia no puede ser empleada como criterio para delimitar el alcance de los principios de justicia sólo a los contextos donde previamente ya exista tal estructura. Si la justicia exige que la interacción social se realice en términos justos, entonces los principios de justicia serán de aplicación en cualquier espacio donde exista interacción social. De acuerdo con Forst (2011: 14), debemos distinguir entre las instituciones que sirven como instrumentos para realizar la justicia, como es el caso de la estructura básica según la interpretación de Freeman, de las circunstancias que hacen la justicia necesaria, como sería la existencia de interacciones, prácticas o instituciones que producen dominación o explotación. Las teorías relacionales de justicia tienen por finalidad analizar las estructuras sociales tal como existen y permitirnos juzgar en base a sus características objetivas si estas son injustas o no. Tal juicio viene acompañado por una exigencia moral: las injusticias deben ser eliminadas en la medida de lo posible con las herramientas de las que en cada momento dispongamos. Las disquisiciones de tipo pragmático que se propondrían al señalamiento de los posibles caminos para resolver tales injusticias o los instrumentos adecuados para modificar las estructuras sociales en cada contexto dado corresponden 
a otro nivel de reflexión, cuyas particulares dificultades en nada cancelan el juicio objetivo previo sobre la injusticia de las estructuras sociales tal como existen y han sido creadas. Si en el contexto global no existiera ninguna "estructura básica» que colaborase en la realización de la justicia y esta fuese instrumentalmente necesaria para lograr tal fin, entonces lo que la justicia exige sería crearla. Freeman, al defender que la existencia previa de tal estructura es necesaria para que las reivindicaciones de justicia sean legítimas, confunde lo que es una exigencia de la justicia con una precondición suya.

Se podría también señalar que la interpretación que del Estado o de la sociedad hace la concepción cooperativista de Freeman es excesivamente simplificadora. Según esta concepción, sólo cada sociedad particular constituye un sistema de cooperación social, y es sólo porque participan en tal sistema que los miembros de la sociedad se deben recíprocamente justica distributiva. En contra de la perspectiva de Freeman se puede sostener que la sociedad es mucho más que un sistema cooperativo entre individuos para la mutua provisión de bienes (Blake 2001: 289). La sociedad se constituye mediante un sistema estructural que conforma de un modo diferencial y estable las opciones de múltiples tipos disponibles para todos sus integrantes. A un mismo tiempo posibilita diversas opciones vitales y las limita, las hace inteligibles o impensables (Castoriadis 2013: 179). Constituye el marco de desarrollo de sus subjetividades y de la objetividad del mundo. Las dinámicas de cooperación constituyen tan sólo algunas de las relaciones sociales que existen entre sus miembros y son relevantes para determinar algunas de las obligaciones que se dan en la sociedad, pero no pueden totalizarse como las únicas relaciones sociales que imponen obligaciones de justicia.

Finalmente, no sólo las afirmaciones normativas de Freeman son cuestionables, sino también las empíricas, de tal modo que incluso si aceptásemos sus criterios de delimitación de las reivindicaciones legítimas de justicia, tendríamos dificultados para concluir que estas se limitan a las sociedades particulares. No es algo evidente que a nivel global no exista cooperación social ni alguna forma de estructura básica. La concepción que mantiene Freeman del contexto global es notablemente simplificadora. La vieja idea de que en el ámbito internacional está formado exclusivamente por "pueblos" con una voluntad unificada y movidos por sus propios intereses egoístas, tiende ya a ser rechazada dentro de la teoría de las relaciones internacionales. En tal ámbito tiene a verse, más bien, una comunidad internacional, una comunidad política equipada tanto por espacios públicos de discusión en los que generar consensos como por instituciones con poder decisorio que, aunque instituidas por los Estados, son capaces de desarrollar una actividad autónoma (Rodrigo 2016). Ciertamente tales instituciones no conforman un Estado global, pero tampoco se debería presuponer que toda 
estructura básica en relación a la cual quepa demandar justicia tenga que ser semejante a la de los Estados nacionales.

Freeman enumera los elementos que componen la estructura básica de la sociedad:

the political constitution; the legal system of trials and other legal procedures it supports; the institution of property; markets and the myriad laws and convention making economic production, exchange, and consumption possible; and the institution of the family, which enables a society to raise and educate children and reproduce itself as an ongoing system over time (Freeman 2006:

38. Nota 16).

Pero Freeman no prueba que únicamente si contamos con todos estos y sólo estos elementos, y sólo del modo como existen en el contexto nacional, estamos ante una estructura social que regula de tal modo la cooperación que puede haber reivindicaciones legítimas de justicia. Además, no resulta difícil señalar instituciones globales que correspondan con cada uno de estos elementos de la estructura básica. Incluso para los que podrían resultar más dudosos, como la existencia de una constitución política a nivel global, no parece justificada la afirmación de Freeman de que esta no podría existir (id., 39). Por un lado, no sería extravagante considerar que el conjunto de textos legales que regulan el orden global (tratados y convenios internacionales, la Carta de las Naciones Unidas, etc.) pudiera realizar un papel similar al de las constituciones nacionales y, por otro, como ha defendido Habermas (2006), la constitucionalización de la sociedad mundial sería plenamente posible e incluso podría considerarse como una condición para su legitimación. Incluso en el caso de que no existiera en la actualidad, tal constitucionalización de la sociedad global debería por lo tanto ser vista, no como una condición previa para que pudieran existir reivindicaciones de justicia legítimas dentro de tal sociedad, sino como una exigencia de la justicia, un instrumento que debe ser creado para que los intercambios sociales sean justos.

\section{Sangiovanni}

Sangiovanni quiere restringir las reivindicaciones igualitaristas de justicia distributiva al marco estatal, pero en contra de Nagel o Blake no cree que el criterio de la coerción sirva para apoyar este objetivo (Sangiovanni 2007: 13-19; 2012: 83-90). Está de acuerdo en que la coerción estatal exige una justificación especial ante sus propios ciudadanos, pero cuestiona que tal justificación deba necesariamente realizarse a través de un principio de justicia distributiva igualitaria como mantienen tales pensadores (Sangiovanni 2012: 
90-107). En su opinión, el criterio buscado debería estar más directamente en conexión con la cuestión de una igual distribución de bienes, mientras que la coerción estatal puede justificarse mediante otras consideraciones no necesariamente distributivas, como por ejemplo haciendo referencia a la libertad o seguridad que promueve. Sangiovanni cree que específicamente las demandas distributivas igualitarias tienen sentido dentro de un tipo concreto de relaciones sociales caracterizadas por "a requirement of reciprocity in the mutual provision of a central class of collective goods, namely those goods necessary for a developing and acting on a plan of life" (Sangiovanni 2007: 4). Dado que su objetivo es restringir estas demandas a los ciudadanos de un mismo Estado, el siguiente paso es obvio:

\footnotetext{
because states (except in cases of occupied or failed states) provide these goods rather than the global order, we have special obligations of egalitarian justice to fellow citizens and residents, who together sustain the state, that we do not have respect to noncitizens and nonresidents (id.).
}

Esto, sin embargo, no significa, en contra de Nagel, que a nivel global no exista ningún tipo de obligación de justicia distributiva, sino que estas no son de tipo igualitario. Según la concepción relacional de la justicia, los principios de justicia particulares que pretenden aplicarse a algún tipo de institución social dependen de qué sea lo que esta hace. En nuestro caso tenemos que preguntarnos qué es lo que hace un Estado a diferencia de lo que ocurre en el orden institucional global (id. 19). Según Sangiovanni, el Estado suministra a sus ciudadanos los bienes colectivos que aseguran las condiciones necesarias para desarrollar y actuar según sus planes de vida. Estas condiciones son la protección frente a ataques físicos y el mantenimiento de un sistema de propiedad (id. 19-20). Los ciudadanos o residentes en un mismo Estado se deberían entre sí justicia distributiva igualitaria por la reciprocidad que se deben las personas que hacen posible esta provisión del Estado. A continuación, se expondrán algunas dificultades de esta propuesta.

Al igual que Freeman, Sangiovanni resalta la relevancia moral de la provisión común de bienes de cierto tipo, razón por la cual comparte algunos de los problemas señalados en el apartado anterior. Al justificar las obligaciones de justicia en base a una idea de reciprocidad según la cual "others are owed a fair return for what they have given you" (Sangiovanni 2007: 26), se excluye a quienes no pueden participar en la provisión común de bienes o incluso que la distribución deba ser igualitaria o no parecería depender de lo que cada cual puede efectivamente hacer. Esta propuesta no concebiría obligaciones de justicia con, por ejemplo, personas con discapacidades severas. Además, en nuestro mundo en globalización, no parece que a partir del criterio de Sangiovanni podamos concluir, como él pretende, que sólo entre ciudadanos o residentes de 
un mismo Estado son legítimas las demandas de justicia distributiva igualitaria. Estas sólo son legítimas entre los que efectivamente participan en la común provisión de ciertos bienes, pero muchas personas que no residen en un mismo Estado participan de algún modo en cualquier provisión (Armstrong 2009: 306). El criterio de la participación en un esquema para la provisión de bienes necesarios para desarrollar nuestros planes de vida parecería llevarnos más bien a una concepción de la justicia que no depende de ámbitos geográficos concretos ni de estatus de ciudadanía. Si a pesar de esto Sangiovanni quiere seguir manteniendo la restricción de la justicia distributiva igualitaria al marco del Estado, debe ofrecer una explicación mucho más compleja de qué significa contribuir en la provisión de tales bienes y en qué sentido esta contribución sólo se da dentro de cada Estado.

Según este autor, los principios de justicia distributiva son igualitarios en virtud de la reciprocidad que se deben las personas que participan en lo que Moellendorf llama "common good associations", asociaciones cuya finalidad es producir «goods and powers to which no one has a preassociational moral claim" (Moellendorf 2011). Sangiovanni considera el Estado como una asociación para producir los bienes necesarios para mantener los sistemas de seguridad y propiedad, pero ¿por qué sólo un sistema para producir estos bienes puede dar origen a principios distributivos igualitarios? No resulta difícil aceptar que los agentes implicados por igual en la producción de tales bienes tienen un derecho igual sobre ellos que no tienen quienes no participan en tal producción, pero ¿no ocurre lo mismo en cualquier otra asociación para producir otros bienes? Imaginemos un empresario que establece una relación contractual con ciertos empleados con el fin de producir beneficio en forma de capital y no seguridad o un sistema de propiedad. Resultaría difícilmente aceptable que por el simple hecho de que los empleados se encontrasen en un país diferente al del empresario no sería exigible que entre ellos hubiese reciprocidad y no se repartiesen de un modo igualitario el bien producido colectivamente, mientras que en el caso de encontrarse en el mismo país sí sería exigible. Recordemos que la igualdad no exige estrictamente que todos reciban de hecho lo mismo, sino que, a partir de una base de referencia de igualdad estricta, cualquier desviación debe estar justificada por razones moralmente relevantes, de modo que, según la clásica justificación del capitalismo, resultaría aceptable desde una concepción igualitarista que el capitalista recibiera mayores beneficios que sus empleados debido a su aporte de capital y los mayores riesgos asumidos. En cualquier caso, estas consideraciones nos llevan a dudar de que el criterio de la reciprocidad debida en este tipo de asociaciones pueda fundamentar la idea de que la justicia distributiva igualitaria sólo tiene sentido dentro del marco de cada Estado. Parecería más bien una exigencia aplicable en cualquier asociación y la forma final que adopte la distribución justa dependerá de los 
bienes específicos que se produzcan en la asociación, sus fines y el modo en que esta se encuentre organizada.

No obstante, posiblemente Sangiovanni no interpreta la justicia distributiva igualitaria como se ha expuesto en el párrafo anterior, como igualdad respecto a la distribución de los bienes concretos producidos en una asociación. Los bienes considerados en el caso del Estado tienen importancia porque constituyen las condiciones necesarias para desarrollar los planes de vida que cada cual se propone. La igualdad no se referiría por lo tanto a la distribución de ningún bien en concreto, sino a la obligación compartida por todos los ciudadanos o residentes del Estado para que todos y cada uno de ellos puedan desarrollar sus planes de vida. Pero en esta interpretación también nos encontramos con dificultades. Según ella, la justicia distributiva igualitaria es exigible sólo entre quienes producen los bienes necesarios para desarrollar el propio plan de vida. Aquí la cuestión crítica es si el establecimiento y el mantenimiento de los sistemas de seguridad y de propiedad por parte de los ciudadanos o residentes en un Estado y sólo ellos es condición suficiente para poder desarrollar el propio plan de vida (Armstrong 2009: 308). No parece difícil imaginar muchas otras condiciones necesarias para poder realizar este fin. Sangiovanni habla de la necesidad de un sistema de propiedad para desarrollar nuestros planes de vida, pero a lo que parece más bien referirse es a la necesidad de producir y distribuir bienes materiales necesarios para nuestra vida. Más que de un sistema de propiedad habría que hablar más en general del sistema económico, el cual está constituido por más elementos que un sistema de propiedad (id.). Además, es cuestionable que los dos sistemas que menciona Sangiovanni sean todo lo que necesitamos para desarrollar nuestros planes de vida. Este autor lo afirma dogmáticamente como si se tratase de alguna evidencia, pero no es difícil imaginar otras condiciones imprescindibles para nuestras vidas. Armstrong (id. 309) enumera algunas: bienes ambientales, infraestructuras, un sistema de reproducción cultural, un sistema político, etc. Y la cuestión aquí es si realmente podemos afirmar que en nuestro mundo contemporáneo en globalización son sólo los ciudadanos o residentes de un Estado los que colaboran en la creación y mantenimiento de los sistemas que garantizan tales bienes.

Del mismo modo que ocurría en la propuesta de Freeman, en el caso actual no sólo nos topamos con problemas normativos sino también empíricos. Incluso aceptando que para nuestros planes de vida sólo necesitamos seguridad y un sistema de propiedad, parece difícil sostener que sólo nuestros conciudadanos o residentes en nuestro mismo Estado colaboran en hacer realidad tales condiciones. Parecen más bien bienes que requieren una colaboración o producción global. Si atendemos a la seguridad, no podemos dejar de señalar que tradicionalmente los Estados han buscado reducir costes a través de acuerdos internacionales que buscan la colaboración en la provisión 
de tal bien. En este sentido, existen organizaciones no sólo militares como la OTAN, sino también de cooperación policial y judicial como la INTERPOL. Además, se puede añadir que acuerdos y organizaciones de este tipo no han surgido simplemente con la finalidad de reducir costes, sino como la única forma de responder eficazmente en nuestro actual mundo en globalización a los nuevos riesgos que debemos afrontar. De acuerdo con Walzer (2006: xiv), podemos extraer una lección sobre seguridad colectiva de los conflictos bélicos acaecidos desde la Primera Guerra del Golfo: "if measures short of war are to work against evil or dangerous regimes, they have to be the common work of a group of nations. They require multilateral commitment. Collective security must be a collective project". Sangiovanni cree que el nivel estatal y el global se distinguen decisivamente en que éste presupone y depende de aquél, en el sentido de que involucra a Estados, no a ciudadanos (Sangiovanni 2007: 21). Sin embargo, aunque los acuerdos internacionales sean suscritos por Estados, la labor de actuar para garantizar la seguridad recae finalmente, al igual que ocurre en el contexto estatal, sobre personas concretas (jueces, policías, militares, etc), de modo que es difícilmente aceptable que estas no colaboren para producir tal bien. En cualquier caso, incluso si aceptásemos la afirmación de Sangiovanni de que los actores del marco global son Estados, no ciudadanos, parecería seguirse, en contra de lo que él quiere defender, que al menos entre ellos existen reivindicaciones igualitarias legítimas de justicia distributiva.

En segundo lugar, el sistema de propiedad o más en general el sistema económico está conformado a través de una compleja red de legislación, acuerdos, relaciones, etc. que trascienden el marco estatal. Esta red se conforma y mantiene a través de un sistema institucional estable que incluye regulaciones sobre patentes y derechos de autor, la Organización Internacional del Trabajo, la Organización Mundial de Comercio, etc. No sólo los Estados tienen presencia en estas instituciones, como prueba la existencia de mecanismos a través de los cuales personas pueden demandar a Estados diferentes a los suyos o a sus ciudadanos. Los intercambios que se producen al interior de un Estado, no sólo en el marco trasnacional, son posibles gracias a todas estas instituciones. Como diversos sociólogos insisten en la actualidad, ya no parece posible trazar una clara división entre lo nacional y lo global. En el mundo globalizado actual, "el predominante concepto de una dualidad global/nacional» no responde adecuada o útilmente a la relación entre economía global y Estado nacional (Sassen 2001: 25). El "nacionalismo metodológico", la perspectiva analítica que trata al Estado como el espacio que contiene los bienes y funciones que conforman cada sociedad particular, constituye una desfiguración insostenible de mundo contemporáneo (Sager 2016; Wimmer y Schiller 2002). 


\section{Abizadeh y Miller}

Al tratar la propuesta de Freeman se sugirió que la interacción, más que su concepción de cooperación social, parecía un criterio más adecuado para señalar los límites de la justicia. Este criterio permitiría desarrollar una concepción de justicia relacional no limitada a priori al marco estatal y capaz de señalar sujetos específicos de justicia para cada contexto (Caney 2008: 493-494). Sin embargo, como se tratará de mostrar a continuación, esta idea es engañosa y distorsiona el objeto de análisis de la justicia en los contextos trasnacionales. Uno de sus problemas queda de manifiesto en la desacertada crítica que Abizadeh (2007) dirige a la propuesta de Freeman. A través de esta crítica su autor no pretende simplemente rechazar esta propuesta, sino que apunta más allá, a la idea general de la justicia relacional de que las reivindicaciones de justicia son coextensivas y relativas a las instituciones que median las relaciones entre los sujetos. Abizadeh rechaza que el "site" de la justicia, según su terminología las instituciones a las que se aplican los principios de justicia, sea coextensivo con su "scope", los sujetos con reivindicaciones legítimas (Abizadeh 2007: 323). El problema de la interpretación de Abizadeh se encuentra en su concepción del "site", al reducirlo arbitrariamente a un subconjunto «básico» y restringido de instituciones que además entiende como algo prescindible y meramente instrumental. En pocas palabras, guiado por la perspectiva interaccional, no es capaz de comprender el peso ontológico de la estructura social.

Abizadeh parte de la "división de trabajo» propuesta por Rawls (Rawls 2002: 84-87). Por un lado, tendríamos el conjunto de las interacciones sociales y, por otro, la "estructura básica", un conjunto de instituciones que pueden o no estar presentes para cualquier conjunto de interacciones que consideremos (Abizadeh 2007: 331-332). La estructura básica desempeña un papel simplemente instrumental, es un medio para lograr que en tal conjunto de interacciones se cumplan ciertas condiciones sociales, pero sin invadir excesivamente la libertad de acción, intercambio, contratación, etc. de los actores individuales implicados. Tal como Rawls indicaba, incluso suponiendo que tanto las condiciones sociales de partida como los acuerdos han sido suscritos libremente, con el paso del tiempo la mala suerte o las malas decisiones terminarán creando desigualdades que hacen imposible la igualdad de oportunidades o la igualdad política, o suponer que los más desfavorecidos establecen acuerdos de forma realmente libre. Para impedir que se llegue a esta situación, según Abizadeh sería conveniente servirse de una estructura básica "in order to realice justice in the system of social interaction and to make it a fair system of social cooperation" (id., 332). De acuerdo con esta interpretación, la división de trabajo propuesta por Rawls establece una distinción entre, por un lado, el conjunto de las interacciones realizadas entre los agentes sociales 
$\mathrm{y}$, por otro, un conjunto de instituciones, la estructura básica, que puede existir o no, y cuya función es conseguir que en tal conjunto de interacciones se den ciertas condiciones.

No se discutirá aquí si esta es una interpretación correcta de la propuesta de justicia de Rawls, aunque mi opinión es que no, sino que sólo se señalará que malinterpreta profundamente lo que es un sistema social. No existe sociedad sin estructura social, de la que el conjunto de instituciones que arbitrariamente consideremos como básico constituye simplemente un subsistema (Bunge 2000; Sewell 1992). Es decir, cualquier interacción social está fundamentada sobre una estructura social y esta no es algo que pueda ponerse o quitarse a conveniencia, sino un componente necesario de toda sociedad. La estructura social hace referencia a un conjunto de instituciones que pueden encontrarse más o menos formalizadas, legalmente o no, prácticas, normas y recursos (Sewell 1992) que conforman tanto simbólica como materialmente la realidad social estableciendo los canales de acción posibles y pensables. En virtud de ella no todo es igualmente posible o pensable para los diferentes grupos sociales. Si se denuncia la injusticia de una sociedad, con ello no se dice que en ella no exista una estructura que conforme de cierto modo las interacciones, sino que la estructura que necesariamente existe, básica o no, conforma las interacciones de un modo injusto. La "división de trabajo" propuesta por Rawls tenía por finalidad señalar diferentes ámbitos de evaluación moral. Por una parte, las interacciones en sí mismas evaluables desde la ética tradicional y, por otro, la estructura social, el objeto de la evaluación que se hace desde la teoría de la justicia. Lo relevante para esta es la estructura, necesariamente presente en todo sistema social y que es reproducida mediante el conjunto de las interacciones sociales. Esta no es un mero instrumento que se pone o se quita como un medio para un fin, sino un componente básico de la ontología social que constituye el objeto de evaluación de la teoría de la justicia. En resumen, empleando la terminología de Abizadeh, el "site", entendido como la estructura social conforma cualquier conjunto de interacciones, y el «scope" no son ciertamente lo mismo, pero son coextensivos. En función de las estructuras que se consideren, las concretas obligaciones de justicia y los agentes implicados serán distintos.

Otro problema de la perspectiva interaccional queda de manifiesto en Globalizing Justice, de Richard W. Miller (2010). En este caso se observa que esta perspectiva favorece el ocultamiento de la estructura que subyace a las interacciones. Si el análisis se centra simplemente en las interacciones de los agentes, en si estos obran bien o mal, si abusan o no del poder que tienen, se pierde de vista el modo como la estructura favorece sistemáticamente a algunos agentes en perjuicio de otros. Se ocultan los mecanismos gracias a los cuales los agentes han adquirido su poder y pueden usarlo abusivamente 
o no, o se presentan unas acciones y no otras como posibles cursos de acción (Haslanger 2015). En esta obra, Miller recurre a la perspectiva interaccional con la finalidad de escapar de los límites estatistas de la justicia: "interactions across borders are the real basis for a vast, unmet transnational responsibility" (Miller 2010: 2). De acuerdo con este autor, estas responsabilidades incumplidas surgen del abuso que de su poder trasnacional hacen los agentes poderosos, a través del cual se aprovechan del estado de necesidad de otros agentes, en especial Estados pobres. En este esquema no hay lugar para los patrones sistémicos que producen esa distribución de poder y de necesidad. Miller señala cuatro interacciones relevantes a nivel trasnacional: explotación en la economía trasnacional, desigualdad en los acuerdos de comercio internacional, negligencia en los daños climáticos e irresponsabilidad imperial. Para todos estos casos, las obligaciones de justicia se basan en el deber de compensación por el uso impropio que individuos, empresas y gobiernos en los países desarrollados hacen de la desesperada necesidad de las personas en los países en desarrollo (id. 59). Estos abusos se realizan a través de los acuerdos y contratos que aquellos agentes imponen a estos para favorecer sus propios intereses, provechándose consciente e intencionalmente del desigual poder de negociación que existe entre ellos.

Este planteamiento muestra una simplificación completamente insostenible de las interacciones globales al plantear por un lado agentes necesitados en países en desarrollo y, por otro, agentes poderosos dispuestos a abusar en los países desarrollados. El problema que interesa resaltar aquí es, sin embargo, que plantear las responsabilidades simplemente como algo interaccional, basadas en la compensación debida por acciones concretas reprochables, oculta las responsabilidades que las personas tienen por las estructuras que comparten y explican sus diversas circunstancias (Young 2011: 107-131). El problema que esta perspectiva plantea a la propuesta de Miller consiste en que el daño que se hace a las personas y respecto al cual cabe reclamar justicia depende de una estructura social que no se crea ni reproduce necesariamente mediante decisiones explícitas ni la intención de abusar por parte de los agentes implicados. Generalmente,

the individual agents involved, far from intending to participate in the production of these effects, are scarcely even aware that they have done so. Their vision is obscured by the seeming naturalness of the choices presented to them, by the independent character of their own reasons for acting as they do, by the complexity of the larger processes to which their actions contribute, by the often minute contribution to those processes made by any single action considered individually, and by the phenomenological priority of individual over group (Scheffler 2001: 44). 
Los graves problemas de justicia a los que nos enfrentamos al considerar los complejos procesos trasnacionales no pueden ser abordados críticamente ignorando tales procesos y simplemente exigiendo que cada agente particular actúe correctamente y de buena fe en sus interacciones particulares. Desde la perspectiva de la justicia no son las acciones personales las que son evaluadas, sino el trasfondo estructural que determina cómo esas acciones de relacionan y condicionan las formas de vida de todos.

\section{Conclusión}

La reducción típicamente rawlsiana de justicia a justicia distributiva pesa enormemente en el marco de análisis y en las respuestas de los teóricos de justicia analizados en este artículo. También en sus preocupaciones de partida. Es notable su desinterés por otras reivindicaciones de justicia relevantes y legítimas relacionadas, siguiendo la ya clásica distinción de Fraser, con el reconocimiento y la dominación. A todos ellos les mueve el interés por justificar una mayor preocupación por el bienestar material de los propios conciudadanos $\mathrm{o}$, mejor, que los recursos ganados esforzadamente por una comunidad política no deban gastarse en personas que, como se supone, no han desempeñado ningún papel relevante en la generación de tales recursos. Las simplificaciones de las que adolecen frecuentemente los debates sobre justicia global y contra las que nos alertan los análisis desarrollados en este artículo, son realmente problemáticas. Sin embargo, como se insistirá al final, estos límites rígidos $\mathrm{y}$ simplificaciones no son problemas que pertenezcan necesariamente a la concepción relacional de la justicia. Es posible otra forma de interpretarla que nos provea de herramientas verdaderamente críticas frente a las injusticias observables en cualquier marco, nacional o global.

La estrategia que siguen los autores analizados aquí para sostener que sólo existen obligaciones de justicia distributiva entre conciudadanos de un mismo Estado consiste en señalar relaciones sociales de cierto tipo que sólo existan entre tales sujetos. Sin embargo, esta forma de argumentar sólo puede conducirnos a la conclusión buscada a través de premisas tan fuertes que resultan poco plausibles. En concreto, presentando lo que satisfactoriamente podría aceptarse como condiciones suficientes para fundamentar reivindicaciones de justicia como implausiblemente las únicas necesarias (Abizadeh 2007: 354-355). Este problema es evidente en las estrategias que siguen Freeman y Sangiovanni. El primero defiende que sólo ante la presencia de una estructura básica caracterizada por incorporar cierto conjunto determinado de elementos se da la cooperación social requerida para imponer obligaciones de justicia distributiva, pero no explica por qué todos y sólo esos elementos son los únicos 
necesarios para configurar una estructura relevante desde el punto de vista de la justicia distributiva. Un problema que se magnifica al considerar que para cada ítem se puede encontrar alguna correspondencia institucional en la esfera global, por lo que una segunda cuestión sin justificar es por qué esos elementos son sólo relevantes si adoptan la forma que muestran en nuestros espacios nacionales y no de ningún otro modo. Por su parte, Sangiovanni fundamenta las exigencias de justicia distributiva en la cooperación en torno a la producción de un conjunto restringido de bienes (la seguridad y un sistema de propiedad), sin justificar por qué necesariamente esos y sólo esos bienes y en presencia del mismo problema de que la provisión de esos bienes no se restringe al espacio nacional. La forma de argumentar de estos pensadores relacionales parece $a d$ hoc: conocen de entrada la conclusión y buscan algunos rasgos diferenciales que distingan a los conciudadanos de los extranjeros. El precio de este recorrido es enorme, pues reducen las relaciones relevantes de la justicia a las caracterizadas por tales rasgos y sólo por ellos. La teoría de la justicia pierde toda su fuerza crítica, escorada hacia la simple apología chovinista.

Pero esta no es una deriva necesaria de la concepción relacional. Podemos seguir a Iris Marion Young y rechazar la restricción arbitraria que Rawls y muchos de sus seguidores hacen de la estructura social a evaluar desde el punto de vista de la justicia a algún subconjunto "básico" de instituciones (Young 2011: 61-88). Desde una perspectiva más general, si cualquier rasgo estructural impone relaciones evaluables desde la justicia, cualquiera de ellos puede ser un foco de injusticia, ya sean las prácticas informales que perseveran en nuestros espacios domésticos, las normas o valoraciones sociales transmitidas por los medios de comunicación, los mecanismos legales de imposición y redistribución económica, o los procedimientos formalizados de representación y decisión políticos. La teoría de la justicia, por lo tanto, perseguiría la injusticia desde "a contextual starting point, for it traces the actually existing relations of positive rule and domination and exploitation, of structural asymmetries and arbitrary rule" (Forst 2011: 15). Cualquier relación social puede, por lo tanto, dar pie a reivindicaciones y obligaciones legítimas, pero no se presupone de qué tipo de obligaciones se trata. Pueden ser distributivas o de otro tipo, o siendo distributivas no se presupone de qué modo habrá de resolverse la injusticia implicada.

Esta perspectiva rechaza que la justicia deba necesariamente reducirse a una de dos perspectivas excluyentes: o bien las reivindicaciones legítimas sólo existen entre ciudadanos o residentes de un mismo Estado, o bien todas las personas se deben lo mismo en base a su común humanidad. La cuestión de si un determinado problema de justicia pertenece al marco estatal o algún otro no puede establecerse a priori. Para responder a ella será necesario un cuidadoso análisis de las relaciones sociales de las que emerge el problema. 
Sin duda, las relaciones que se establecen entre los ciudadanos de un Estado son especialmente densas y se encuentras reguladas de forma especialmente profunda por instituciones comunes, por lo que este marco continuará constituyendo un caso muy importante para la teoría de la justicia. Pero lo importante es insistir en que constituye un caso entre otros (Cohen y Sabel 2006: 149). El objeto de evaluación de la justicia son estructuras sociales concretas y cada una de ellas puede generar injusticias específicas e imponer obligaciones diferentes.

No resulta apropiado imaginar lo estatal o lo global como contextos homogéneos, tal como tienen a hacer los pensadores "estatistas" analizados aquí, así como sus críticos "cosmopolitas". De acuerdo con Tan, por poner un ejemplo de estos últimos, la injusticia fundamental a nivel global surge del hecho de que las personas ricas se asocian instituyendo Estados cuyo objetivo es defender sus intereses y aprovechar su mayor poder de negociación frente a otros Estados constituidos por personas pobres, exacerbando así la desigualdad preexistente (Tan 2006: 342-343). Se ha llegado a convertir en un lamentable lugar común interpretar la injusticia global simplemente como Estados ricos de ciudadanos ricos que rehúyen sus obligaciones frente a Estados pobres de ciudadanos pobres. Sin embargo, en un momento histórico en el que las redes, conexiones y espacios sociales a nivel global se complejizan enormemente, la vieja división del mundo entre "Primer" y "Tercer" Mundo, países pobres y ricos, se revela como una peligrosa desfiguración ideológica de la realidad (Mezzadra 2005: 20; Mezzadra y Neilson 2017: 47-82). Las relaciones sociales de cualquier marco de justicia deben ser analizadas desde aproximaciones más matizadas, haciéndose cargo de la complejidad del objeto de análisis. Contextos de "Tercer mundo" conviven juntos o incluso superpuestos a otros de "Primer mundo". Dos frecuentes presuposiciones acerca de la ciudadanía han dejado de ser sostenibles en el mundo contemporáneo, si es que alguna vez lo fueron: su congruencia con un Estado o nación y su homogeneidad en torno a la característica de clase (Faist 2015: 26). Esto nos señala que deberíamos evitar sacar conclusiones apresuradas de simples datos macroeconómicos referentes a países como totalidades unificadas. En contra de Tan, el problema de la justicia global no es que en el origen haya desigualdad y el conjunto de los ricos se asocie conformando Estados, lo cual tenga como resultado la exacerbación de las desigualdades originarias. El problema son las diversas y complejas relaciones estructurales, institucionalizadas de diversas formas y en grados diversos, que explican tanto las desigualdades de partida como especialmente su mantenimiento y reproducción. Las cuales no sólo operan favoreciendo sistemáticamente a algunos en la distribución de recursos, sino también atribuyendo a algunos grupos sociales mayor estimación social o estatus, o confiriéndoles mayor poder en la toma de decisiones vinculantes. 
En la actual fase de la globalización, las relaciones sociales son demasiado complejas como para seguir ubicándolas necesariamente en un único marco de justicia. El problema ya no es que debamos elegir entre estar de acuerdo con los estatistas o con los globalistas, sino más bien que no podemos asumir a priori que todos los problemas de justicia pertenecen o a un marco estatal o a uno global. Es más, no se trata simplemente de que algunas injusticias se puedan analizar correctamente como perteneciendo a un marco estatal mientras que otras a uno global, sino de que un mismo problema de injusticia puede que pertenezca simultáneamente a varios marcos de justicia. En estos casos, resulta fundamental analizar cómo dinámicas estructurales pertenecientes a lo nacional y a lo global o local se refuerzan mutuamente. Los problemas de injusticia no son sólo interseccionales en relación a las dimensiones de distribución, reconocimiento y representación, sino también en relación a los marcos o escalas estructurales desde los que es preciso analizarlos (Fraser 2010: 364). Los mecanismos sociales generadores de injusticia son sistemas complejos de poder, dominación o explotación, que pueden involucrar a muy diferentes instituciones y agentes que actúan en diferentes ámbitos: instituciones familiares o religiosas, autoridades locales o regionales, gobiernos, corporaciones multinacionales, organismos internacionales, etc. Una teoría de la justicia debería, por lo tanto, poder abordar las injusticias en toda su complejidad: "the various contexts of justice -local, national, international, and global- are connected through the kind of injustice they produce, and a theory of justice must not remain blind to this interconnectedness» (Forst 2001: 167). 


\section{Bibliografía:}

Abizadeh, Arash, Cooperation, Pervasive Impact, and Coercion: On the Scope (not Site) of Distributive Justice, Philosophy \& Public Affairs, 35 (4), 318-358, (2007).

Armstrong, Chris, Coercion, Reciprocity, and Equality Beyond the State, Journal of Social Philosophy, 40 (3), 297-316, (2009).

Blake, Michael, Distributive Justcie, State Coercion, and Autonomy, Philosophy \& Public Affairs, 30 (3), 257-296, (2001).

Bunge, Mario, Systemism: the alternative to individualism and holism, Journal of Socio-Economics, 29), 147-157, (2000).

Caney, Simon, Global Distributive Justice and the State, Political Studies, 56), 487-518, (2008).

Castoriadis, Cornelius, La institución imaginaria de la sociedad (Ciudad de México: Tusquets, 2013).

Cohen, Joshua, y Sabel, Charles, Extra Rempublicam Nulla Justitia?, Philosophy \& Public Affairs, 34 (2), 147-175, (2006).

Faist, Thomas, Migración y teorías de la ciudadanía. En P. Mateos (Ed.). Ciudadanía múltiple y migración : perspectivas latinoamericanas (2556). (Ciudad de México: CI-DE/CIESAS2015).

Forst, Rainer, Towards a critical theory of transnational justice, Metaphilosophy, 32 (1/2), 160-179, (2001).

Forst, Rainer, Transnational justice and democracy, Normative orders, 04/2011), (2011).

Fraser, Nancy, Injustice at Intersecting Scales: on "Social Exclusion" and the "Global Poor", European Journal of Social Theory, 13 (3), 363-371, (2010).

Freeman, Samuel, The Law of Peoples, Social Cooperation, Human Rights, and Distributive Justice, Social Philosophy and Policy, 23 (1), 29-68, (2006).

Habermas, Jürgen, ¿Una constitución política para una sociedad mundial pluralista? Entre naturalismo y religión (315-355). (Barcelona: Paidós2006).

Haslanger, Sally, What is a (social) structural explanation? Springer Science+Business Media, doi:10.1007/s11098-014-0434-5 (2015).

Mezzadra, Sandro, Derecho de fuga. Migraciones, ciudadanía y globalización (Madrid: Traficantes de sueños, 2005).

Mezzadra, Sandro, y Neilson, Brett, La frontera como método (Madrid: Traficantes de sueños, 2017).

Miller, Richard W., Globalizing Justice. The Ethics of Poverty and Power (Oxford: Oxford University Press, 2010). 
Moellendorf, Darrel, Cosmopolitanism and Compatriot Duties, The Monist, 94 (4), 535-554, (2011).

Nagel, Thomas, El problema de la justicia global, Revista Jurídica de la Universidad de Palermo, 9 (1), 169-196, (2008).

Rawls, John, La justicia como equidad. Una reformulación (Barcelona: Paidós, 2002).

Rawls, John, Teoría de la Justicia (Ciudad de México: Fondo de cultuta económica, 2014).

Rodrigo, Ángel J., Entre Westfalia y Worldfalia: la comunidad internacional como comunidad social, política y jurídica. En C. García Segura (Ed.). La tensión cosmopolita. Avances y límites en la institucionalización del cosmopolitismo (23-63). (Madrid: Tecnos2016).

Sager, Alex, Methodological Nationalism, Migration and Political Theory, Political Studies, 64 (1), 42-59, doi: https://doi.org/10.1111/14679248.12167 (2016).

Sangiovanni, Andrea, Global Justice, Reciprocity, and the State, Philosophy \& Public Affairs, 35 (1), 3-39, (2007).

Sangiovanni, Andrea, Justice and the Priority of Politics to Morality, The Journal of Political Philosophy, 16 (2), 137-164, (2008).

Sangiovanni, Andrea, The Irrelevance of Coercion, Imposition, and Framing to Distributive Justice, Philosophy \& Public Affairs, 40 (2), 79-110, (2012).

Sassen, Saskia, ¿Perdiendo el control? La soberanía en la era de la globalización (Barcelona: Bellaterra, 2001).

Scheffler, Samuel, Individual Responsibility in a Global Age Boundaries and Allegiances: Problems of Responsibility and Justice in Liberal Thought. (Oxford: Oxford University Press2001).

Sewell, William H., A Theory of Structure: Duality, Agency, and Transformation, American Journal of Sociology, 98 (1), 1-29, (1992).

Tan, Kok-Chor, The Boundary of Justice and the Justice of Boundaries: Defending Global Egalitarianism, Canadian Journal of Philosophy, XIX (2), 319-344, (2006).

Walzer, Michael, Just and Unjust Wars. A Moral Argument woth Historical Illustrations $4^{\mathrm{a}}$ ed. (Nueva York: Basic Books, 2006).

Wimmer, Andreas, y Schiller, Nina Glick, Methodological nationalism and the study of migration, European Journal of Sociology, 43 (2), 217-240, (2002).

Young, Iris Marion, Responsabilidad por la justicia (Madrid: Morata, 2011). 